\title{
Adverse effects and drug interactions of medications commonly used in the treatment of adult HIV positive patients
}

\author{
Rachel Heylen, Robert Miller
}

\section{Introduction}

Patients with advanced HIV disease commonly take a large number of medications that are active against HIV, that treat or prevent opportunistic infections and that prevent the symptoms of HIV itself or those produced by the other medications. Many of these medications have overlapping toxicities, and share the same methods of elimination from the body, leading to varied drug interactions.

This article seeks to highlight those drugs that are used in HIV infected patients which are most commonly associated with drug interactions, the reasons for these interactions and to help physicians and pharmacists make decisions about which of the interactions are clinically significant. By examining available data on the toxicity, formulation and pharmacokinetics of these different medications charts and explanatory notes have been compiled.

\section{Pharmacodynamic Interactions (tables 1-4)}

Additive toxicity One or a number of the main side effects of the two drugs overlap and can lead to increased toxicity. The classification of this toxicity is denoted by a letter:

$$
\begin{aligned}
& \text { M-Myelotoxicity } \\
& \text { P-Pancreatitis } \\
& \text { N-Peripheral neuropathy } \\
& \text { K-Nephrotoxicity } \\
& \text { L-Hepatotoxicity }
\end{aligned}
$$

The clinical significance of this interaction is as below.

Pharmacy University College London Hospitals (NHS) Trust, Middlesex Hospital Site, London, UK R Heylen

Department of Sexually Transmitted Diseases, Division of Pathology and Infectious Diseases, University College London Medical School and Camden and Islington Community Health Services (NHS) Trust, London, UK R Miller

Address correspondence to: Dr R F Miller, 4th Floor, Mortimer Market Centre, Mortimer Market, (off Capper Street), London WCIE 6AU, UK. Accepted for publication 26 June 1996
Increased Serum Levels This is usually due to inhibition of metabolism by the liver or excretion via the kidneys, and can lead to increased toxicity. The drug that has been affected is indicated by the direction of the arrow (table 1). The clinical significance of this interaction is described below.

Decreased Serum Levels This is often caused by an increase in metabolism by the liver as a result of the use of drugs which induce liver enzymes such as rifampicin, or by a decrease in the absorption of the drug from the gastrointestinal tract such as the bioavailability of ketoconazole is reduced by the administration of drugs which increase gastric $\mathrm{pH}$. The clinical significance of this interaction is as below.

\section{Miscellaneous}

Interactions that have been reported but which do not fall under the above headings.

\section{Clinical Significance}

The interactions have been allocated to one of four categories as follows:

?-unknown clinical significance This category is used when there have been reports in the literature of an interaction but these are only case reports or in vitro work or the conclusions of these reports are conflicting. It also includes drugs where an interaction may be expected (from those reported with other members of the same class of drugs etc) but no reports have been found, this may be important for drugs that have been recently released on to the market.

! - take note This category is used when there is evidence of an interaction but it is of theoretical interest rather than clinical importance or it involves the fine tuning of dosing eg reducing the dose of a relatively non toxic medication in renal failure.

!! - use with caution This category is used when there is evidence of an interaction, and it is of clinical importance. Adjustments may have to be made in doses of toxic medications etc.

skull and cross-contraindicated The combination of drugs should be avoided where possible. This includes well documented interactions that are clinically significant, combinations that are precluded by the manufacturer or combinations which share potentially very serious side effects, for example nephrotoxicity with co-administration of IV amphotericin and IV pentamidine.

Antiretrovirals: Zidovudine (AZT), didanosine (ddI), zalcitabine (ddC), and stavudine (D4T) (Table 1)

These four medications are nucleoside analogues which prevent viral replication by either causing chain termination and/or blocking the reactive site of the enzyme reverse transcriptase thus inhibiting retroviral cDNA synthesis. They share some similarities in that all are metabolised in infected cells to the active triphosphate form. They are metabolised to differing extents by the liver, and both changed and unchanged drug is excreted in the urine.

\section{Pharmacokinetic interactions}

Approximately $70 \%$ of zidovudine is metabolised in the liver by glucuronidation to 3azido-3-deoxy-5-D-glucopyranosyl thymidine. Both changed and unchanged drug are excreted in the urine by renal tubular excretion. ${ }^{1}$ After 





oral administration of didanosine, $50 \%$ is excreted via the kidneys by active tubular secretion and glomerular filtration. The metabolism of didanosine has not been evaluated in man. Studies in dogs show that it undergoes extensive hepatic metabolism. ${ }^{2}$ Zalcitabine is excreted largely unchanged in the urine. ${ }^{3}$ The excretion of stavudine is divided between renal and non renal routes, undergoing net renal tubular secretion. ${ }^{4}$

Zidovudine has the greatest potential for interactions with drugs that influence liver metabolism. Many drugs have been suspected of inhibiting the metabolism of zidovudine..$^{5}$ In an early study the concurrent use of paracetamol (not in the table) with zidovudine was associated with an increased incidence of myelotoxicity, ${ }^{6}$ and a case of severe hepatotoxicity has been reported. ${ }^{7}$ These were thought to be caused by a pharmacokinetic interaction between zidovudine and paracetamol, however further research has not shown changes in the pharmacokinetic parameters of the two drugs. ${ }^{89}$

Clarithromycin has been reported both to reduce zidovudine concentrations and to have no effect. ${ }^{11}{ }^{11}$ Similarly, serum levels of phenytoin have been reported to be both increased and decreased when zidovudine is started. ${ }^{5}$ Methadone has been shown to increase the area under the curve (AUC) of zidovudine in some patients. ${ }^{12}$ and In vitro and in vivo work which has examined the effect of the azoles on zidovudine metabolism, has shown a decrease in metabolism of zidovudine to it's glucuronide. The clinical significance of this remains uncertain. ${ }^{13}$ Concomitant administration of probenecid may inhibit the metabolism of zidovudine to the glucuronide metabolite and may also decrease the rate of excretion of zidovudine and its metabolite. ${ }^{14}$ This may also occur with didanosine and stavudine. ${ }^{154}$

Rifabutin used as prophylaxis of Mycobacterium avium-intracellulare (MAI) has been shown to decrease zidovudine $C_{\max }$ and AUC, and increase zidovudine clearance. ${ }^{16}$ It is likely that rifampicin would do likewise.

Few drugs have been reported to alter the pharmacokinetics of didanosine, zalcitabine and stavudine. Oral ganciclovir increases plasma levels of didanosine by an unknown mechanism. ${ }^{17}$ The combination of IV ganciclovir and didanosine may increase the potential for pancreatitis but the reason for this has not been elucidated. ${ }^{18}$

The renal excretion of zidovudine, didanosine, zalcitabine and stavudine may be inhibited by drugs that cause renal toxicity such as aminoglycosides, IV amphotericin, foscarnet, cidofovir and IV pentamidine. Dosage reductions are recommended by the manufacturers for zidovudine, didanosine, and stavudine in patients with renal impairment. The dose of zalcitabine should also be reduced but neither the degree of reduction nor information about when this reduction should take place is available.

Didanosine is unstable at the $\mathrm{pH}$ found in the stomach. Therefore it is supplied as a chewable/dispersible tablet with dihydroxyaluminium sodium carbonate, magnesium hydroxide and sodium citrate as buffer to increase the $\mathrm{pH}$ of the stomach and thus its bioavailability. The presence of the buffer may interfere with absorption of a number of drugs by two different mechanisms. By increasing the $\mathrm{pH}$ it may reduce the solubility and thus absorption of acid soluble drugs eg dapsone, ketoconazole, itraconazole, pyrimethamine, trimethoprim and rifampicin. ${ }^{19}$ Alternatively, the aluminium and magnesium ions contained in the chewable/dispersible tablet formulation may bind (chelate) the drug molecules eg ciprofloxacin, and tetracyclines (not in the table) thus preventing their absorption. ${ }^{2}$ To reduce the effect of didanosine on the absorption of other drugs they should be administered at least 2 hours apart.

\section{Pharmacodynamic interactions}

The most frequent adverse effects after chronic use of zidovudine are related to bone marrow toxicity and result in anaemia and neutropenia. Those of didanosine and zalcitabine are peripheral neuropathy and pancreatitis. Zalcitabine has also been reported to cause thrombocytopenia in approximately $4 \%$ of patients. The major dose limiting toxicities of stavudine are peripheral neuropathy and increased hepatic transaminases. ${ }^{4}$ The combined use of ganciclovir and zidovudine has been reported to be poorly tolerated in patients with HIV and serious cytomegalovirus (CMV) disease. In one study $82 \%$ of patients on this combination developed severe to life threatening haematological complications. ${ }^{20}$ Zidovudine should be stopped whilst patients are receiving high dose intravenous ganciclovir. It may be reintroduced (generally at a lower dose) when the dose of ganciclovir is reduced, or the patient is transferred to the oral formulation.

The combination of foscarnet with zidovudine is haematologically better tolerated than that of ganciclovir and zidovudine. However, one small study has reported increased transfusion.requirements in patients receiving both zidovudine and foscarnet. This occurred mainly during the treatment period. ${ }^{21}$ Care should be taken with other drugs reported to cause haematological toxicity (see table 1, opposite page).

Drugs that cause peripheral neuropathy should be used with caution in combination with didanosine, zalcitabine, or stavudine and drugs that cause pancreatitis in combination with didanosine and zalcitabine. If this is not possible in an acute clinical situation, then the antiretroviral medication should be stopped whilst treatment is taking place and restarted after a one-to-two week wash out period. ${ }^{22}$ If chronic treatment is required the patient should be closely monitored for clinical and biochemical signs of pancreatitis or peripheral neuropathy.

\section{Miscellaneous}

Zidovudine and Aciclovir There is a single report of a patient who whilst receiving intravenous aciclovir for the treatment of herpes simplex infection experienced severe lethargy 


\begin{tabular}{|c|c|c|c|c|}
\hline ANTI FUNGAL DRUGS & $\begin{array}{l}\text { Ampho } \\
\text { tericin }\end{array}$ & $\begin{array}{l}\text { Keto/Itra } \\
\text { conasole }\end{array}$ & $\begin{array}{c}\text { Flu } \\
\text { conasole }\end{array}$ & $\begin{array}{c}\text { Flucyto } \\
\text { sine }\end{array}$ \\
\hline Alcohol & & $?$ & & \\
\hline Aminoglycosides & & & iิ! & $\hat{\imath} ! !$ \\
\hline Amphotericin (IV) & & $?$ & $?$ & 仓!! \\
\hline Antacids & & $\diamond 0$ & & \\
\hline Astemizole, terfenadine, cisapride & & $\Leftrightarrow \&$ & 42 & \\
\hline Atovaquone & & & & \\
\hline Azithromycin & & & & \\
\hline Carbamazepine & & $\nLeftarrow ! !$ & $\Leftrightarrow !$ & \\
\hline Cidofovir + probenecid & 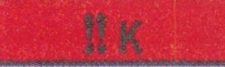 & & î! & î!! \\
\hline Ciprofloxacin & s! & & & \\
\hline Corticosteriods & $!$ & $\Leftrightarrow !$ & & \\
\hline Clarithromycin & $\Leftrightarrow !$ & & & \\
\hline Dapsone & 2928 & & & 32 \\
\hline Didanosine (ddl) & ६!!! & $\hat{\mathrm{u}} \mathrm{O}$ & & \\
\hline Doxo/Daunorubicin (liposomal) & 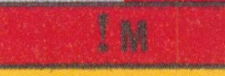 & & & \\
\hline Fluconazole & $?$ & & & \\
\hline Flucytosine & $\leftrightarrow 1$ & & & \\
\hline Food & & $\hat{I}$ & & \\
\hline Foscarnet & & & i! ! & î!! \\
\hline Ganciclovir & $<!$ & & & 138 \\
\hline Isoniazid & 34 & $\hat{\imath} !$ & & \\
\hline Itra/Ketoconazole & $?$ & & & \\
\hline Methadone & & & & \\
\hline Metronidazole & & & & \\
\hline Oral Contraceptive & & & & \\
\hline Pentamidine (IV) & 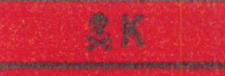 & & $\hat{\mathrm{u} !}$ & $\hat{\imath} ! !$ \\
\hline Phenytoin & 24 & $\diamond ! ! \Uparrow$ & $\leqslant$ !! 代 & \\
\hline Pyrimethamine & $\sqrt{11}$ & & & 13 \\
\hline Rifabutin & & $\leftrightarrow ! ! \hat{\imath}$ & $\hookleftarrow ! ! \Uparrow$ & 811 \\
\hline Rifampicin & & पे & 介ि!! & 2010 \\
\hline Stavudine (D4T) & \&! 14 & & & \\
\hline Sulphonamides (high dose) & II $\mathrm{x}$ KX & & & IIIt \\
\hline Thalidomide & 14? & & & 138 \\
\hline Vinblastine / Vincristine & $? 3$ & $\leftarrow !$ & ६? & 31 \\
\hline Zalcitabine (ddC) & $29<$ \&!! & & & 132 \\
\hline Zidovudine (AZT) & 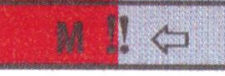 & \&? & ६? & 4172 \\
\hline $\begin{array}{l}\text { Additive Toxicity } \\
\text { Increased Serum Levels } \\
\text { Decreased Serum Levels } \\
\text { Miscellaneous }\end{array}$ & \multicolumn{2}{|c|}{$\begin{array}{l}\text { M- Myelotoxicity } \\
\text { P - Pancreatitis } \\
\text { N - Peripheral Neuropathy } \\
\text { K - Nephrotoxicity } \\
\text { L- Hepatotoxicity }\end{array}$} & \multicolumn{2}{|c|}{$\underset{\substack{\text { Increasing Clinical } \\
\text { Significance }}}{\stackrel{?}{\stackrel{2}{x}}}$} \\
\hline
\end{tabular}


and fatigue on initiation of zidovudine. These symptoms resolved when the aciclovir was stopped..$^{23}$ In practice zidovudine and aciclovir are frequently used together without this problem being encountered.

Zidovudine and pyrimethamine and pyrimethamine/sulphadiazine Zidovudine has been found to antagonise the toxoplasmocidal effect of a low concentration of pyrimethamine in vitro, and in vitro synergism between pyrimethamine and sulphadiazine against Toxoplasma gondii is reversed by zidovudine. Zidovudine also antagonises the therapeutic effect of pyrimethamine in mice acutely infected with $T$ gondii. The clinical significance of this finding is unclear. ${ }^{24}$

Zidovudine and stavudine In cell cultures zidovudine inhibits the phosphorylation of stavudine, while stavudine has no effect on the phosphorylation of zidovudine. This finding may potentially influence the decision to use stavudine and zidovudine as combination therapy for HIV infection. ${ }^{25}$

Zidovudine/didanosine and ganciclovir In vitro work has suggested that ganciclovir antagonises the anti-HIV effect of zidovudine/didanosine. ${ }^{26}$

Didanosine and drugs with a low bioavailability such as atovaquone and oral ganciclovir The buffer in didanosine may induce diarrhoea in some patients. In patients taking drugs with a low bioavailability this may reduce absorption and make the medication ineffective. ${ }^{27}$

\section{Antifungal medication: amphotericin, ketoconazole, itraconazole, fluconazole and flucytosine (Table 2 see opposite page)}

Antifungal medications are widely used in the treatment of the HIV positive patient. Severity of infection ranges from superficial oral candida to cryptococcal meningitis and pulmonary aspergillosis. The sensitivity of the organism, site of infection, drug toxicity and interactions will often dictate the choice of therapy to be used. Amphotericin, although extremely nephrotoxic is the treatment of choice in severe infections.

\section{Pharmacokinetic interactions}

Ketoconazole, and itraconazole share many of the same interactions. They require an acid environment for optimal absorption, are extensively metabolised in the liver and excreted almost exclusively in the faeces and the urine. In contrast the absorption of fluconazole is unaffected by gastric $\mathrm{pH}$ and it is minimally metabolised, with $80 \%$ being excreted unchanged in the urine. Fluconazole is the only azole for which dose reduction is necessary in renal impairment and care needs to be taken with nephrotoxic drugs.

All three azoles inhibit the cytochrome P450 liver metabolising isoenzyme CYP3A4 leading to increased serum levels of many drugs (see table 2). ${ }^{28-31}$ Concurrent administration of azoles with astemizole, terfenadine and cisapride is contraindicated following reports of prolongation of the QT interval and fatalities. ${ }^{29} 32$
Phenytoin toxicity has been observed when fluconazole was added to patients' treatment regimens. $^{33}$

The metabolism of ketoconazole, itraconazole, and fluconazole is accelerated by drugs which induce liver enzymes such as rifampicin, rifabutin, phenytoin etc. Almost negligible serum levels of itraconazole have been reported in a patient taking concurrent rifampicin and itraconazole, ${ }^{3435}$ and poor treatment outcomes and clinical relapse have been reported in patients taking fluconazole and rifampicin. ${ }^{36}{ }^{37}$ Carbamazepine and phenobarbitone have also been shown to induce itraconazole metabolism. ${ }^{38}$ Isoniazid decreases plasma levels of ketoconazole and so may possibly have the same effect on itraconazole levels. ${ }^{39}$

The absorption of ketoconazole and itraconazole is reduced as the $\mathrm{pH}$ of the stomach increases. In a study comparing the absorption of ketoconazole and fluconazole in patients receiving intravenous cimetidine, in order to titrate their stomach acid to $\mathrm{pH} 6.0$ and above, there was a dramatic effect on the disposition of ketoconazole. Reductions of $90 \%$ in AUC and maximum concentrations were seen..$^{40}$ The disposition of fluconazole was unaffected. To increase the solubility and thus absorption of ketoconazole and itraconazole patients are advised to take them with an acidic carbonated beverage eg lemon and lime carbonated drinks, carbonated water or cola. ${ }^{41}$ Any drug which increases gastric $\mathrm{pH}$ including didanosine, should be taken at least two hours after ketoconazole or itraconazole, and where possible they should be taken with or after food (except for itraconazole liquid which contains a buffer and is better absorbed in a fasted state).

Flucytosine is excreted mostly unchanged in the urine. It is extremely myelotoxic and toxicity is related to serum levels. Serum levels must be monitored closely especially when it is being used in combination with a nephrotoxic agent eg amphotericin.

\section{Pharmacodynamic interactions}

The main toxicities of amphotericin are fever and chills on administration, abnormal renal function, hypokalaemia and hypomagnesaemia. Acute liver failure, normochromic normocytic anaemia, thrombocytopenia, leucopenia and other haematological abnormalities have also been reported. The use of amphotericin with other nephrotoxic drugs should be avoided wherever possible or if this is not possible it should be used with extreme caution (see table 2). Acute, rapidly progressive, renal failure has been documented with the simultaneous use of IV amphotericin and IV pentamidine. ${ }^{42}$

The main toxicities of the azoles are rash and elevations of liver function tests. Unless abnormal liver function tests are present the use of azoles with other potentially hepatotoxic drugs is not precluded. There is a possible interaction between alcohol and ketoconazole (see under miscellaneous).

As flucytosine is extremely myelotoxic, its use with other myelotoxic drugs should be avoided. If this is not possible eg the use of flucytosine with cytotoxic drugs, or IV ampho- 


\begin{tabular}{|c|c|c|c|c|}
\hline MYCOBACTERIAL DRUGS & Rifampicin & Rifabutin & Clarithro & Azithro \\
\hline \multicolumn{5}{|l|}{ Alcohol } \\
\hline Aminoglycosides & & $\hat{\imath} !$ & 1ิ! & \\
\hline Amphotericin (IV) & & $\hat{\imath} !$ & $\hat{\imath} !$ & \\
\hline Antacids & tิ? & & & 10 \\
\hline Astemizole, terfenadine, cisapride & & & 2 & \&? \\
\hline Atovaquone & 58 & \&? & & \\
\hline Azithromycin & & 仓!!! & & \\
\hline Carbamazepine & & & ↔!! & \\
\hline Cidofovir + probenecid & & i! ! & 仓! & \\
\hline \multicolumn{5}{|l|}{ Ciprofloxacin } \\
\hline Corticosteriods & ৫!! & «? & ६! & \\
\hline Clarithromycin & $\Leftrightarrow !$ & 介!! \&! & & \\
\hline Dapsone & $\Leftrightarrow ! !$ & & & \\
\hline Didanosine (ddl) & î? & & & \\
\hline Doxo/Daunorubicin (liposomal) & $7 \mathrm{M}$ & 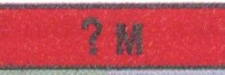 & & \\
\hline Fluconazole & $\leftrightarrow ! !$ & ↔! 介!! & & \\
\hline Flucytosine & 2118 & 385 & & \\
\hline Food & $\hat{1} 0$ & & & $\hat{i} \theta$ \\
\hline Foscarnet & & î! & $\hat{\imath} !$ & \\
\hline Ganciclovir & 284 & ?III & & \\
\hline \multicolumn{5}{|l|}{ Isoniazid } \\
\hline Itra/Ketoconazole & स्थें & « !! $\hat{\imath}$ & & \\
\hline Methadone & $\Leftarrow !$ & «? & & \\
\hline \multicolumn{5}{|l|}{ Metronidazole } \\
\hline Oral Contraceptive & $\measuredangle ! !$ & $\leftrightarrow !$ & & \\
\hline Pentamidine (IV) & & ㄴ! & $\hat{\imath} !$ & \\
\hline Phenytoin & $\Leftrightarrow ! !$ & $\Leftrightarrow ?$ & & \\
\hline Pyrimethamine & $? M$ & $? \mathrm{M}$ & & \\
\hline Rifabutin & & & «!! 仓! & «!! 仓̂? \\
\hline Rifampicin & & & 仓!!! & 仓ิ? \\
\hline \multicolumn{5}{|l|}{ Stavudine (D 4T) } \\
\hline \multicolumn{5}{|l|}{ Sulphonamides (high dose) } \\
\hline \multicolumn{5}{|l|}{ Thalidomide } \\
\hline Vinblastine / Vincristine & $? \mathrm{814}$ & $? \mathrm{M}$ & $\leftrightarrow ?$ & \\
\hline \multicolumn{5}{|l|}{ Zalcitabine $(\mathrm{ddC})$} \\
\hline Zidovudine (AZT) & \&? & $\Leftrightarrow ?$ & $\& ?$ & \\
\hline Additive Toxicity & \multirow{4}{*}{\multicolumn{2}{|c|}{$\begin{array}{l}\text { M- Myelotoxicity } \\
\text { P - Pancreatitis } \\
\text { N - Peripheral Neuropathy } \\
\text { K - Nephrotoxicity } \\
\text { L- Hepatotoxicity }\end{array}$}} & \multirow{4}{*}{\multicolumn{2}{|c|}{ 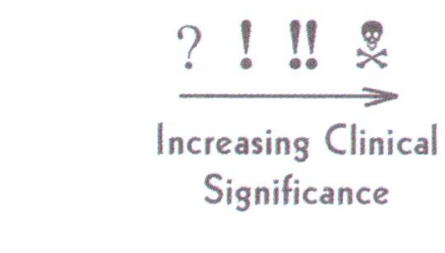 }} \\
\hline Increased Serum Levels & & & & \\
\hline Decreased Serum Levels & & & & \\
\hline Miscellaneous & & & & \\
\hline
\end{tabular}


tericin, close monitoring of the patient's haematological parameters is necessary.

\section{Miscellaneous}

Amphotericin and corticosteroids The combined use of IV amphotericin and corticosteroids may exacerbate the renal potassium loss caused by amphotericin. ${ }^{43}$

Amphotericin and azoles There is a theoretical interaction between amphotericin and azoles. Azoles inhibit ergosterol synthesis and thereby eliminate the target for the activity of amphotericin. The clinical significance of this interaction has not yet been clearly defined. ${ }^{44} 45$

Ketoconazole and alcohol Exceptional cases of a disulfiram-like reaction to alcohol characterised by flushing, rash, peripheral oedema, nausea and headache have been reported. All symptoms resolved completely within a few hours. ${ }^{39}$

\section{Mycobacterial drugs: rifampicin, rifabutin, clarithromycin and} azithromycin (Table 3 see opposite page) Combinations of these drugs are used to treat both Mycobacterium tuberculosis (TB) and disseminated MAI infections.

\section{Pharmacokinetic interactions}

Rifampicin and rifabutin are derivatives of the rifamycin molecule. Both are hepatically metabolised. Metabolic products are principally excreted in bile and to a lesser extent in urine. The dose of rifampicin does not have to be reduced in patients with renal impairment, but the dose of rifabutin should be reduced if the creatinine clearance falls below $30 \mathrm{ml} / \mathrm{min}$. Both drugs induce liver enzymes resulting in the accelerated metabolism of other hepatically metabolised drugs (see table 3), the effect of rifampicin being greater than that of rifabutin. The effects of enzyme induction may be seen within a few days, but maximal induction takes more than a week to occur.

The effect of rifampicin on the metabolism of azoles (see above), corticosteroids, clarithromycin, dapsone, methadone, the oral contraceptive, phenytoin and warfarin (not in table) are clinically very important. For example; it is recommended that the dose of corticosteroids be doubled in some instances. ${ }^{46}$ It is reported that prophylaxis of Pneumocystis carinii pneumonia with dapsone failed because of the interaction with rifampicin ${ }^{47}$ and serum levels of phenytoin, and the clinical effectiveness of warfarin and the oral contraceptive are reduced. ${ }^{484}$ Rifampicin increases the rate of metabolism of many opiates including methadone and withdrawal symptoms may be induced. ${ }^{50}$

In one prospective study, rifampicin, and to a lesser extent rifabutin, were shown to significantly reduce the mean serum levels of clarithromycin. ${ }^{51}$ This has raised doubts as to the effectiveness of this combination for the treatment of MAI and further studies are required. Clarithromycin in turn has been shown to inhibit the metabolism of rifabutin leading to an increased incidence of uveitis and neutropenia. Fluconazole and potentially the other azoles have also been shown to increase plasma levels of rifabutin..$^{52-57}$

Clarithromycin and azithromycin are both macrolide antibiotics. Clarithromycin is extensively hepatically metabolised to its active metabolite 14-hydroxy clarithromycin. The half life of clarithromycin and its metabolite are increased in the presence of renal impairment, leading to the recommendation that the dose is reduced in this situation. The proportion of absorbed azithromycin that undergoes hepatic biotransformation is unknown. Several metabolites have been identified but these are not thought to possess significant antimicrobial activity. The primary route of azithromycin elimination may be via the bile and faeces, although transintestinal elimination has also been proposed as a primary route of elimination. Less than $6 \%$ of a dose of azithromycin is recovered in the urine within one week of administration. ${ }^{58}$

The mechanism of the inhibition of hepatic cytochrome $\mathbf{P 4 5 0}$ enzymes by macrolide antibiotics has been well documented. ${ }^{59}$ Clarithromycin shares many of the interactions of erythromycin. It appears to inhibit the metabolism of carbamazepine leading to increased serum concentrations, ${ }^{60}$ it inhibits the metabolism of the non-sedating antihistamines and cisapride increasing the risk of cardiac arrhymias. ${ }^{61}$ The potential for other interactions seen with erythromycin needs to be borne in mind eg increased levels of digoxin and theophylline, and enhanced clinical effects of corticosteroids warfarin ${ }^{62}$ and vinblastine. ${ }^{63}$

In contrast to clarithromycin, azithromycin has been shown to be relatively free of drug interactions. It does not exhibit the interaction with the non-sedating antihistamines and cisapride ${ }^{61}$ However, the possibilities of interactions should be considered as an increase in neutropenia comparable to that which occurs with clarithromycin was reported when azithromycin was used in conjunction with rifabutin. ${ }^{64}$

\section{Pharmacodynamic interactions}

With all these drugs most frequently encountered side effects involve the gastrointestinal tract, for example nausea and vomiting.

Rifampicin and rifabutin will stain body secretions red/orange colour, rifabutin will also stain the skin. Both have the potential to increase liver enzymes, and to cause neutropenia. High serum concentrations of rifabutin increase the incidence of uveitis and are also associated with a syndrome of arthralgia/ arthritis. ${ }^{52-57}$ In the treatment of TB abnormal liver function tests, that may be rifampicin induced, have been reported more commonly in alcohol abusers, those aged $>35$ years, the malnourished or those with known hepatic disease. Care should be taken with the use of rifampicin in these patients. ${ }^{65}$

\section{Miscellaneous}

Clarithromycin and zidovudine See under zidovudine 


\begin{tabular}{|c|c|c|c|c|}
\hline ANTIVIRALS & Aciclovir & Ganciclovir & Foscarnet & \begin{tabular}{|l}
$\begin{array}{l}\text { Cidofovir \& } \\
\text { probencid }\end{array}$ \\
\end{tabular} \\
\hline \multicolumn{5}{|l|}{ Alcohol } \\
\hline Aminoglycosides & 仓ิ! & ก!!! & 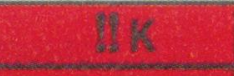 & \\
\hline Amphotericin (IV) & $\hat{\imath} !$ & i! ! & 38 & $3 x$ \\
\hline \multicolumn{5}{|l|}{ Antacids } \\
\hline \multicolumn{5}{|l|}{ Astemizole, terfenadine, cisapride } \\
\hline \multicolumn{5}{|l|}{ Atovaquone } \\
\hline \multicolumn{5}{|l|}{ Azithromycin } \\
\hline \multicolumn{5}{|l|}{ Carbamazepine } \\
\hline Cidofovir + probenecid & !! & 仓!!! & $8 x$ & \\
\hline Ciprofloxacin & & & $?$ & \\
\hline Corticosteriods & & & $?$ & \\
\hline Clarithromycin & & & $\Leftrightarrow !$ & \$! \\
\hline \multicolumn{5}{|l|}{ Dapsone } \\
\hline Didanosine (ddl) & & i!？ & \&!! & ک!! \\
\hline \multicolumn{5}{|l|}{ Doxo/Daunorubicin (liposomal) } \\
\hline Fluconazole & & & ६! & \&! \\
\hline Flucytosine & & 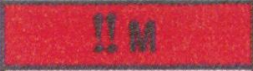 & ६!! & S!! \\
\hline \multicolumn{5}{|l|}{ Food } \\
\hline Foscarnet & 11! !! & 仓ิ!! & & 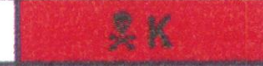 \\
\hline Ganciclovir & !! & & \&!! & $\diamond ! !$ \\
\hline \multicolumn{5}{|l|}{ Isoniazid } \\
\hline \multicolumn{5}{|l|}{ Itra/Ketoconazole } \\
\hline \multicolumn{5}{|l|}{ Methadone } \\
\hline \multicolumn{5}{|l|}{ Metronidazole } \\
\hline \multicolumn{5}{|l|}{ Oral Contraceptive } \\
\hline Pentamidine (IV) & $\hat{\imath} !$ & î!! & 2k & $5 \times$ \\
\hline \multicolumn{5}{|l|}{ Phenytoin } \\
\hline \multicolumn{5}{|l|}{ Pyrimethamine } \\
\hline Rifabutin & & & \&! & ৫! \\
\hline \multicolumn{5}{|l|}{ Rifampicin } \\
\hline Stavudine (D4T) & & & \&!! & s!! \\
\hline Sulphonamides (high dose) & $\mathrm{kx}$ & 118 & $14 x$ & $91 \mathrm{~K}$ \\
\hline Thalidomide & & 24 & & \\
\hline Vinblastine / Vincristine & & 118 & & \\
\hline Zalcitabine (ddC) & & & ६!! & s!! \\
\hline Zidovudine (AZT) & $?$ & 1111 & 1!! ? ? & «!! \\
\hline $\begin{array}{l}\text { Additive Toxicity } \\
\text { Increased Serum Levels } \\
\text { Decreased Serum Levels } \\
\text { Miscellaneous }\end{array}$ & \multicolumn{2}{|c|}{$\begin{array}{l}\text { M- Myelotoxicity } \\
\text { P - Pancreatitis } \\
\text { N- Peripheral Neuropathy } \\
\text { K - Nephrotoxicity } \\
\text { L- Hepatotoxicity }\end{array}$} & \multicolumn{2}{|c|}{$\underset{\substack{\text { Increasing Clinical } \\
\text { Significance }}}{\stackrel{?}{\stackrel{e}{x}}}$} \\
\hline
\end{tabular}


Antivirals: aciclovir, ganciclovir, foscarnet, cidofovir and probenecid (Table 4 see opposite page)

In HIV positive patients aciclovir is used for the treatment and maintenance of herpes simplex virus 1 and 2, and varicella zoster. Ganciclovir, foscarnet, and cidofovir are used mainly for the treatment and maintenance of cytomegalovirus, but also have activity against the herpesviruses.

\section{Pharmacokinetic interactions}

Aciclovir is slowly and incompletely absorbed; its oral bioavailability is approximately $15-30 \% .{ }^{66}$ In infected cells it is converted by virus-encoded thymidine kinase to its monophosphate derivative. Subsequent diphosphorylation and triphosphorylation are catalysed by cellular enzymes. Aciclovir triphosphate inhibits viral DNA synthesis by acting as a substrate for viral DNA polymerases and preventing further DNA chain elongation. Aciclovir is minimally metabolised, and about $85 \%$ is excreted unchanged in the urine through glomerular filtration and renal tubular secretion. Drugs such as probenecid that inhibit renal tubular secretion or resorption may reduce clearance of aciclovir and thus increase plasma concentrations. The dose of aciclovir should be reduced in patients with impaired renal function.

Ganciclovir shares the same enzymatic changes and mode of action as aciclovir. It has poor oral bioavailability with values of $3-4 \cdot 6 \%$ being quoted. ${ }^{67}$ Bioavailability may be increased if ganciclovir is taken with food. ${ }^{68}$ Almost $100 \%$ of ganciclovir is excreted unchanged in the urine. As with aciclovir probenecid may increase plasma levels and so the dose of ganciclovir should be reduced in patients with impaired renal function.

Foscarnet has very poor oral absorption, the serum levels required for activity against CMV and other herpesviruses may only be achieved by intravenous administration. Renal clearance accounts for most of the plasma clearance, however, up to $20 \%$ of a cumulative dose may have been deposited in bone 7 day post infusion. ${ }^{69}$ The dose of foscarnet must be reduced in patients with impaired renal function. As foscarnet may cause nephrotoxicity the doses of renally cleared drugs being used concurrently with foscarnet should be monitored closely (see table 4).

Cidofovir is an acyclic nucleotide analogue. Unlike aciclovir and ganciclovir it does not depend on phosphorylation by viral nucleoside kinases to exert its antiviral effect. Instead, the drug is phosphorylated to its active form by cellular enzymes. In vitro studies have suggested that the resulting active metabolites are cleared slowly from the intracellular space. Approximately $90 \%$ of the intravenous dose was recovered unchanged in the urine in 24 hours. It has been shown that active tubular secretion played a significant role in the clearance of cidofovir. The dose limiting toxicity of cidofovir is nephrotoxicity. The concurrent administration of probenecid has been shown to ameliorate its effect on proximal tubular cells, perhaps by decreasing the initial concen- tration of cidofovir in the cortex of the kidney. ${ }^{70}$ The dose of cidofovir must be reduced in patients with impaired renal function although there are few data available on which to base decisions about dose reductions in relation to specific creatinine clearances. As cidofovir may cause nephrotoxicity the doses of concurrently used renally cleared drugs should be monitored closely (see table 4). As cidofovir is always administered with probenecid, it is important to consider probenecid drug interactions (see table 4).

\section{Pharmacodynamic interactions}

Aciclovir is well tolerated. At high intravenous doses aciclovir has been reported to crystalise in the kidneys. Therefore care should be taken with the use of other potentially nephrotoxic drugs and the dose of aciclovir should be reduced if nephrotoxicity occurs.

The dose limiting toxicities of ganciclovir are neutropenia and thrombocytopenia. Care should be taken in using ganciclovir with other myelotoxic drugs as an increased incidence of myelotoxicity has been reported with the concurrent use of zidovudine and ganciclovir (see above).

Foscarnet and cidofovir are both nephrotoxic. As with amphotericin (see above) concurrent use of other nephrotoxic drugs should be avoided where possible.

\section{Miscellaneous}

Aciclovir and zidovudine See under zidovudine

Combined use of aciclovir with ganciclovir/foscarnet/cidofovir This should not be required as ganciclovir/foscarnet/cidofovir all have activity against herpes viruses. Occasionally, patients experience a break through of herpes when on maintenance doses of these agents. If this occurs aciclovir should be used in the normal manner.

Ganciclovir and imipenem (not in table) Seizures have been reported with this combination (data sheet).

Ganciclovir and zidovudine/didanosine See above.

Foscarnet and ciprofloxacin Seizures have been reported with this combination..$^{71}$

Foscarnet and corticosteroids Amphotericin and foscarnet cause potassium depletion, therefore from the interaction between amphotericin and corticosteroids it follows that corticosteroids may increase the potassium depletion seen with foscarnet.

Part 2 In the second part of this article the newer anti retrovirals will be considered: indinavir, saquinavir, ritonavir and lamivudine (3TC). We acknowledge the help of Ms Sally Hibbert, Senior
Computer Services Technician, Pharmacy Department, UCL Hospitals for her help in the design and compilation of the interaction charts and Jane Rutherford for help with the typing of this manuscript.

1 Blum MR, Liao SH, Good SS, de Miranda P. Pharmacokinetics and bioavailability of zidovudine in humans. Am f Med 1988 85: (suppl 2A) 189-4.

2 ABPI Data Sheet Compendium 1995-96:326-8. Videx.

3 ABPI Data Sheet Compendium 1995-96:1431-3. 1431-3. Hivid. 
4 Dudley M, Graham K, Kaul S, et al. Pharmacokinetics of stavudine in patients with AIDS or AIDS-related complex. F Infect Dis 1992;166:480-5.

5 ABPI Data Sheet Compendium 1995-96:1952-6. Retrovir

6 Fischl MA, Richman DD, Grieco MH, et al. The efficacy of azidothymidine (zidovudine) in the treatment of patients with AIDS and AIDS related complex. A double blind
placebo-controlled trial. $N$ Engl $\mathcal{f}$ Med 1987;317: placebo-cor

7 Shriner $\mathbf{K}$, Bidwell Goetz $M$. Severe hepatotoxicity in a patient receiving both acetaminophen and zidovudine. patient receiving both acet
$A m \mathcal{F} M$ Med $1992 ; 93: 94-6$.

8 Steffe EM, King JH, Inciardi JF, et al. The effect of acetaminophen on zidovudine metabolism in HIV-infected patients. F Acquir Immun Def Synd 1990;3:691-4

9 Burger DM, Meenhorst PL, Koks CHW, Beijnen JH Pharmacokinetics of zidovudine and acetaminophen in a patient on chronic acetaminophen therapy. Ann Pharmacother 1994;28:327-30.

10 Craft MS, Craft JC. Drug interaction between clarithromycin and oral zidovudine in HIV-1 infected patients. Clin Pharmacol Ther 1994;53:163.1-114.

11 Vance E, Watson-Bitar M, Gustavson L, Kazanjian P. Pharmacokinetics of clarithromycin and zidovudine in patients with AIDS. Antimicrob Agents Chemother 1995; 39:1355-60

12 Schwartz EL, Brechbuhl AB, Kahl P, Miller MA, Selwyn PA, Friedland GH. Pharmacokinetic interactions of zidovudine and methadone in intravenous drug-using patients with HIV infection. $f$ Acquir Immun Def Synd 1992;5:619-26.

13 Asgari M, Back DJ. Effect of azoles on the glucuronidation of zidovudine by human liver UDP-glucuronosyltransferase. $\mathcal{F}$ zidovudine by human liver

14 Hansten PD, Horn JR. Drug Interactions and Updates. Vancouver, Applied Therapeutics Inc. 1990

15 Jones M, Bristol-Myers Squibb Pharmaceuticals Ltd Personal communication, didanosine and probenecid.

16 Narang P, Nightingale S, Manzone C, et al. Does rifabutin affect zidovudine disposition in HIV + ve patients ? VII International Conference on AIDS, III STD World Congress July 1992; Abstract PoB 3888

17 Gaines K, Wong R, Jung D, et al. Pharmacokinetic interactions with oral ganciclovir: zidovudine, didanosine, probenecid. 10th International Conference on AIDS:

1994 Aug 7-12; Yokohama (Japan) 1994 Abstract 004B.
18 Personal communication Syntex Pharmaceuticals Ltd didanosine and ganciclovir

19 Metroka CE, McMechan MF, Andrada R, Laubenstein LJ, Jacobus DP. Failure of prophylaxis with dapsone in patients taking dideoxyinosine. $N$ Engl $f$ Med 1991; 325:737

20 Hochster H, Dieterich D, Bozette S, et al. Toxicity of Combined ganciclovir and zidovudine for cytomegalovirus disease associated with AIDS. Ann Int Med 1990; 113:111-7

21 Palestine AG, Polis MA, De Smet MD, et al. A randomised controlled trial of foscarnet in the treatment of cytomegalovirus retinitus in patients with AIDS. Ann Int Med 1991;115:665-73.

22 NIAID updates dideoxyinosine toxicity profile, suggests ways to reduce risk of pancreatitis. Clinical Pharmacy ways to reduce
$1990 ; 9: 832-8$.

23 Bach MC. Possible drug interaction during therapy with azidothymidine and acyclovir for AIDS. $N$ Engl $f \mathrm{Med}$ 1987;316:547

24 Israelski DM, Corrynne T, Remington JS. Zidovudine antagonises the action of pyrimethamine in experimental infection with Toxoplasma gondii. Antimicrob Agents Chemother 1989;33:30-4.

25 Sandstrom E, Oberg B. Antiviral therapy in human immunodeficiency virus infections. Current status (part 1). Drugs 1993;45:488-508.

26 Medina DJ, Hsiung GD, Mellors JW. Ganciclovir antagonises the anti-human immunodeficiency virus type activity of zidovudine and didanosine in vitro. Antimicrob Agents Chemother 1992;36:2217-30.

27 Hughes W, Leoung G, Kramer F, et al. Comparison of atovaquone $(566 \mathrm{C} 80)$ with trimethoprim-sulphamethoxazole to treat PCP in patients with AIDS. New Engl f Med 1993;328:1521-7.

28 Seaton TL, Celum CL, Black DJ. Possible potentiation of warfarin by fluconazole. DICP. Ann Pharmacother 1990, 24:1177-8.

29 Honig PK, Wortham DC, Hull R, Zamani K, Smith JE, Cantilena LR. Itraconzole affects single-dose terfenadine pharmacokinetics and cardiac repolarization pharmacopharmacokinetics and cardiac repolarization

30 Bohme A, Ganser A, Hoelzer D. Aggravation of vincristineinduced neurotoxicity by itraconazole in the treatment of induced neurotoxicity by itraconazole in the

31 Olkkola KT, et al. Midazolam should be avoided in patients receiving the systemic antimycotics ketoconazole or itraconazole. Clin Pharmacol Ther 1994;55:481-5.

32 Committee on Safety of Medicines. Medicines Control Agency. Curr Probl Pharmacovigil 1996;22:1-2.

33 Cadle RM, Zenon GJ, Rodriguez-Barradas MC, Hamill RJ. Fluconazole induced symptomatic phenytoin toxicity. Ann Pharmacother 1994;28:191-5.

34 Blomley $M$, Teare EL, de Belder A, Thway $Y$, Weston $M$. Itraconazole and anti-tuberculosis drugs. Lancet 1990; 336:1255

35 Drayton J, Dickinson G, Rinaldi MG. Coadministration of rifampicin and itraconazole leads to undetectable levels of serum itraconazole. Clin Infect Dis 1994;18:266.

36 Nicolau DP, Crowe HM, Nightingale CH, Quintiliani R. Rifampicin-fluconazole interaction in critically ill patients. Ann Pharmacother 1995;29:994-6.

37 Coker RJ, Tomlinson DR, Parkin J, Harris JRW, Pinching AJ. Interaction between fluconazole and rifampicin. $B M \mathcal{F}$
1990;301:818

38 Bonay M, Jonville-Bera AP, Diot P, Lemarie E, Lavandie M, Autret E. Possible interaction between phenobarbital, carbam

39 ABPI Data Sheet Compendium 1995-96:734-5. Nizoral.

40 Blum RA, D'Andrea DT, Florentino BM, et al. Increased gastric $\mathrm{pH}$ and the bioavailability of fluconazole and gastric $\mathrm{pH}$ and the bioavailability of fluconazo

41 Sugar EF, Sugar AM, Kreger BE. Effect of common beverages on the dissolution of ketoconazole tablets. AIDS 1992:6:1221-2.

42 Antoniskis D, Larsen RA. Acute progressive renal failure with the simultaneous use of amphotericin $B$ and pentamidine. Antimicrob Agents Chemother 1990;34:470-2.

43 ABPI Data Sheet Compendium 1995-6:1774 Fungizone intravenous.

44 Pahls S, Schaffner A. Aspergillus fumigatus pneumonia in neutropenic patients receiving fluconazole for infection due to Candida species: is amphotericin B combined with fluconazole the appropriate answer? Clin Infect Dis 1994; 18:484-5.

45 Scheven $M$, Scheven ML. Interaction between azoles and amphotericin B in the treatment of candidiasis. Clin Infect Dis 1995;20:1079.

46 Buffington GA, Dominguez $\mathrm{JH}$, Piering WF, et al. Interaction of rifampicin and glucorticoids. $\mathcal{F} A M A 1976$ 236: $1958-60$.

47 Horowitz HW, Jorde UP, Wormser GP. Drug interactions in use of dapsone for Pneumocystis carinii prophylaxis. Lancet 1992;339:747.

48 Wagner JC. Rifampicin-Phenytoin drug interaction. Drug Intell Clin Pharm 1984;18:497[Abstract]

49 ABPI Data Sheet Compendium 1995-6:950-2. Rifadin.

0 Jacobson M, Yajko D, Nothfelt $\mathrm{D}$, et al. Randomised placebo controlled trial of rifampicin, ethambutol, and ciprofloxacin for AIDS patients with disseminated MAC infection. F Infect Dis 1993;168:112-9.

51 Wallace RJ, Brown BA, Griffith DE Girard W, Tanaka K. Reduced serum levels of clarithromycin in patients treated with multidrug regimens including rifampicin or rifabutin for Mycobacterium avium-M. intracellulare infection. $\mathcal{F}$ Infect Dis 1995;171:747-50.

52 Griffith DE, Brown BA, Girard WM, Wallace RJ. Adverse events associated with high-dose rifabutin in macrolidecontaining regimens for the treatment of Mycobacterium avium complex lung disease. Clin Infect Dis 1995;21: 594-8.

53 Siegal FP, Eilbott D, Burger H, et al. Dose-limiting toxicity of rifabutin in AIDS-related complex: syndrome of arthralgia/arthritis. AIDS. 1990;4:433-41.

54 Rifabutin prophylaxis against Mycobacterium avium complex infection. New Engl f Med 1994;330:436-39. Misc authors. Comments on Nightingale SD, Cameron DW, Gordin FM, et al. Two controlled trials of rifabutin prophylaxis against Mycobacterium avium complex infection phylaxis against Mycobacterium avium co

55 Frank MO, Graham MB, Wispelway B. Rifabutin and uveitis. N Engl f Med 1994;330:868.

56 Fuller ID, Stanfield LED, Craven DE. Rifabutin prophylaxis and uveitis. N Eng $\mathcal{F} M e d$ 1994;330:1315-6.

57 Narang PK, Trapnell CB, Schoenfelder JR, Lavelle JP, Bianchine JR. Fluconazole and enhanced effect of rifabutin prophylaxis. $N$ Engl $\mathcal{F}$ Med 1994;330:1316.

58 Ballow C, Amsden GW. Azithromycin: The first azalide antibiotic. Ann Pharmacother 1992;26:1253-61.

59 Pensayre D, Larrey D, Funck-Brentano C, Benhamen JP. Drug interaction and hepatitis produced by som macrolide antibiotics. F Antimicrob Chemother 1985 16:(Suppl A):181-94.

60 Albani F, Riva R, Barmzzi A, et al. Clarithromycin-carba mazepine interaction: a case report. Epilepsia 1993;34: $161-2$

61 Hansten P, Horn JR. Drug interactions with non-sedating antihistamines. Drug Interactions Nevwsletter 1994:737-40.

62 ABPI Data Sheet Compendium 1995-6:15-6. Klaricid.

63 Tobe SW, Sui LL, Jamal SA, et al. Vinblastine and erythromycin: an unrecognised serious drug interaction.

64 Yonren S. Phase 1 study to evaluate the pharmacokinetic interaction between rifabutin and the macrolides azithromycin and clarithromycin in healthy subjects. azithromycin and clarithromycin in healthy

65 Caplin $M$, Thompson N, Hamilton $M$, McIntyre N, Burroughs A. Antituberculous therapy and acute liver failure. Lancet 1995;345:1171.

66 de Miranda P, Blium MR. Pharmacokinetics of aciclovi after intravenous and oral administration. $f$ Antimicrob Chemother 1983; (Supp1 B):29-37.

67 Jacobson M, de Miranda P, Cederberg DM, et al. Human pharmacokinetics and tolerance of oral ganciclovir Antimicrob Agents Chemother 1987;31:1251-4.

68 Byrne AK, Lavelle J, Follensbee S, et al. Multiple dos cross over pharmacokinetic study to evaluate the effect of food on the relative bioavailability of oral gancicloyir (abstract no. PuB 7077). Proceedings of the VIII International Conference on AIDS and the III STD World Congress; 1992 Jul 19-24: Amsterdam.

69 Sjovall J, Bergdahl S, Movin G, Ogenstad S, Saarimaki M. Pharmacokinetics of foscarnet and distribution to cerebrospinal fluid after intravenous infusion in patients with HIV. Antimicrob Agents Chemother 1989;33:1023-31. 70 Cundy K, Petty BG, Flaherty J, et al. Clinical pharmacokiinfected patients. Antimicrob Agents Chemother 1995;39. 1247-52.

71 Fan-Harvard P, Sanchorawala V, Oh J, Moser EM, Smith SP. Concurrent use of foscarnet and ciprofloxacin may increase the propensitity for seizures. Ann Pharmacother 1994;28:869-72.

$\stackrel{+}{\circ}$

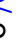

